\title{
Stripping Coupons with Linear Programming
}

\author{
David E. Allen, Lyn C. Thomas, Harry Zheng *
}

\begin{abstract}
When using market prices to fit the parameters of models for the price of bonds, the first step is to strip the market bonds of their coupons. The standard bootstrapping technique of stripping coupons can cause mispricing if there are no bonds that mature for some periods or if there are several bonds that mature at the same time. In this paper we suggest a new linear programming formulation to strip out riskfree and risky zero coupon bond prices, which works whatever the current date, coupon dates, and sampling dates. The stripped US Treasury bond prices match the observed US STRIPS prices. We also discuss issues of liquidity, sampling periods, and implied default probabilities of corporate bonds.
\end{abstract}

\section{Introduction}

When using market prices to fit the parameters of models for the price of bonds, the first step is to strip the market bonds of their coupons. This is because most bond price models really model the current term structures of spot rates of benchmark riskfree and risky securities (Treasury and corporate bonds), that is the prices of zero coupon bonds. However, there are few zero coupon bonds available on the market. Although Treasury STRIPS can be used to represent these theoretical riskfree spot rates, there are some problems with that approach. The main one is that the liquidity of the Treasury strips market is not as great as that of the Treasury coupon market, which means the observed rates on strips reflect a premium for liquidity. So it is necessary to extract spot rates from yields of coupon bonds of different maturities, both in the Treasury and the corporate bond markets.

${ }^{*}$ D.E. Allen is in School of Finance and Business Economics, Edith Cowan University, Western Australia 6027, Australia (d.allen@cowan.edu.au). L.C. Thomas and H. Zheng are in Department of Business Studies, University of Edinburgh, Edinburgh EH8 9JY, UK (l.thomas@ed.ac.uk and h.zheng@ed.ac.uk). The authors are grateful to CDatastream for the data and to Nigel Morkel-Kingsbury for advice on extracting the data. 
The standard methods of stripping coupons are bootstrapping (Fabozzi, 1998) or linear regression (Carleton and Cooper, 1976). If for each period there is one and only one coupon bond that matures, then these techniques generate a unique set of spot rates over the periods. However if there are no bonds that mature for some periods or if there are several bonds that mature at the same time, then there are not unique answers and in some cases the techniques give rise to rates with unacceptable feature, particularly with risky bonds. Jarrow, Lando and Turnbull (1997) for example used these methods to strip out the risky zero coupon bond prices and pointed out several mispricings, such as 5 year AA zero coupon bonds priced above 5 year AAA zero coupon bonds, and 4 year B zero coupon bonds priced below 5 year B zero coupon bonds, etc. The authors attributed these mispricings to the noise of the data and the call features of some bonds.

These mispricings are bothersome because it becomes difficult to estimate the parameters in the credit bond pricing models being developed. There has been a resurgence of interest in such models since they not only give investors a clear indication of current market perceptions of the riskiness of particular bonds, but are also a stepping stone to pricing many credit risky fixed income derivatives, such as callable and putable bonds, caps and floors, MBSs, etc. Jarrow and Turnbull (1998) for example derived the default probabilities of risky bonds by combining a default process with an interest rate model. They applied the Black-Derman-Toy model to build a recombined binomial short rate tree, then combined it with the default process to form a bigger tree for credit risky bonds, and finally obtained default probabilities by forward and backward induction methods.

To remedy the mispricing caused by bootstrapping, Thomas, Allen, and Morkel-Kingsbury (1998) suggested using linear programming to strip out risky zero coupon bond prices. This produces the same spot rates as the bootstrapping technique if there is one and only one coupon bond that matures for each period, but is always able to ensure that for the same maturity the higher rating zero coupon bond is priced above the lower rating zero coupon bond, and for the same credit rating the shorter maturity zero coupon is priced above the longer maturity zero coupon bond. Although difficulties encountered in Jarrow, Lando, and Turnbull (1997) are avoided by the LP formulation, other problems have crept in. The main one is that the gap between zero coupon bond prices of different credit ratings widens and then narrows as time goes by, which means the forward rate of higher credit rated zero coupon bonds is higher than that of lower credit rated zero coupon bonds. Such a behaviour again suggests that there are potential arbitrage opportunities. 
In this paper we suggest a new linear programming formulation to strip out risky zero coupon bond prices, which resolve the problems discussed above. In section two we use an extension of the original LP approach to strip Treasury bonds, which works whatever the current date, coupon dates, and sampling dates. In section three we introduce a new LP formulation for stripping the coupons from risky corporate bonds which ensures that the spreads are increasing over time. Section four discusses how the LP formulation can be modified to deal with liquidity issues and section five looks at an extension to arbitrary time intervals between the sampling points at which the zero coupon bond price is calculated. In section six we discuss the connection between the zero coupon bond prices obtained by the LP formulation and the default probabilities that the market is impugning to the risky corporate bonds.

\section{Treasury STRIPS Prices}

To derive pure discount bond prices $v_{0}(t)$ of riskfree zero coupon bonds paying 1 at a set of prechosen times $t=0,1, \ldots, T$, we use the observed market prices of $N_{0}$ bonds to solve the following linear programming problem:

$$
\begin{aligned}
\text { LP1: minimize } & \sum_{i=1}^{N_{0}}\left(a_{i}+b_{i}\right) \\
\text { subject to } & P_{i}+a_{i}=\sum_{t=1}^{T} c_{i}(t) v_{0}(t)+b_{i} \\
& v_{0}(t) \geq(1+m(t)) v_{0}(t+1) \\
& a_{i}, b_{i} \geq 0
\end{aligned}
$$

for $i=1, \ldots, N_{0}$ and $t=0,1, \ldots, T-1$, where $v_{0}(0)=1, P_{i}$ is the present value of the bond $i, c_{i}(t)$ is its cash flow at time $t$, and $m(t)$ is the minimum expected forward rate from $t$ to $t+1$. The first constraints seek to match the present value $P_{i}$ to the discounted cash flows $c_{i}(t)$ and $a_{i}$ and $b_{i}$ are the mispricing errors. $a_{i}$ is positive and $b_{i}=0$ if the price is "too low"; $b_{i}$ is positive and $a_{i}=0$ if the price is "too high". The second constraint ensures that there is no mispricing with respect to maturity. (In the case when $m(t)=0$ the constraint corresponds to saying bonds of longer maturity should be priced lower than those of shorter maturity.)

The cash flow $c_{i}(t)$ is decided by the coupon payment, coupon date, sampling date, and current date. As an example assume $t_{1}, t_{2}, \ldots, t_{T}$ are fixed semiannual sampling dates. A bond pays a coupon $c$ every six month 
with a principle $F$ and a maturity date before or at time $t_{T}$, then there is one cash flow in each sampling period. Let $v_{i}$ be the price of the riskfree zero coupon bond paying 1 at time $t_{i}$. Assume $\alpha$ is the proportion of the time between a coupon date and the next sampling date compared with the time between two sampling dates (therefore $\alpha$ is a number between 0 and 1 ). Let $\beta$ be the proportion of a sampling interval between the current date (when the market price of the bond is observed) and the next sampling date. There are two cases to consider: 1 . there is no coupon payment between the current date and the next sampling date; and 2. there is one coupon payment.

In the first case, we have $\alpha \geq \beta$ and the relation between the market price and the future cash flows are approximated by the following equation:

$$
\begin{aligned}
P=P_{C}+(\alpha-\beta) c= & \alpha c v_{1}+c v_{2}+\cdots \\
& +c v_{T-2}+(c+\alpha F) v_{T-1}+(1-\alpha)(c+F) v_{T} .
\end{aligned}
$$

Here $P$ is the present value, $(\alpha-\beta) c$ is the accrued interest and $P_{C}$ is the "clean" market price. We have split each coupon payment and the principle into two parts. One $\alpha c$ is paid at the previous sampling date and one $(1-\alpha) c$ is paid at the subsequent sampling date.

In the second case, we have $\alpha<\beta$ and the resulting equation is

$$
\begin{aligned}
P=P_{C}+(1+\alpha-\beta) c= & \left(\frac{\alpha}{\beta}\right) c+\left(\alpha+\frac{\beta-\alpha}{\beta}\right) c v_{1}+c v_{2}+\cdots \\
& +c v_{T-2}+(c+\alpha F) v_{T-1}+(1-\alpha)(c+F) v_{T} .
\end{aligned}
$$

Here $(1+\alpha-\beta) c$ is the accrued interest.

We notice that the two equations are basically the same except for the cash value at the present date and the cash paid out at $t_{1}$. In the case of $\alpha=0$ and $\beta=1$, that is, the current date is the sampling date and the next coupon payment is on the next sampling date, then we have a very simple equation:

$$
P_{C}=c v_{1}+\cdots+c v_{T-1}+(c+F) v_{T} .
$$

This set of timings leads to the special case of LP1 where if bond $i$ has coupon $c_{i}$ and principle $F_{i}$ then

$$
c_{i}(t)=c_{i} \text { for } t=1,2, \ldots, T-1 \text { and } c_{i}(T)=c_{i}+F_{i} .
$$

For more general current dates we have that if $\alpha \geq \beta$ then the cash flows are

$$
c_{i}(1)=\alpha c_{i},
$$




$$
\begin{aligned}
c_{i}(t) & =c_{i} \text { for } t=2, \ldots, T-2, \\
c_{i}(T-1) & =c_{i}+\alpha F_{i} \text { and } \\
c_{i}(T) & =(1-\alpha)\left(c_{i}+F_{i}\right)
\end{aligned}
$$

while for $\alpha<\beta$ with $t_{0}$ being the current date, the cash flows are

$$
\begin{aligned}
c_{i}(0) & =\frac{\alpha}{\beta} c_{i}, \\
c_{i}(1) & =\left(\alpha+\frac{\beta-\alpha}{\beta}\right) c_{i}, \\
c_{i}(t) & =c_{i} \text { for } t=2, \ldots, T-2, \\
c_{i}(T-1) & =c_{i}+\alpha F_{i} \text { and } \\
c_{i}(T) & =(1-\alpha)\left(c_{i}+F_{i}\right) .
\end{aligned}
$$

The proof of equations (2) and (3) is given in the appendix together with other cases.

We used 113 Treasury bonds on February 7, 2000 with maturity dates up to the second half of 2008, whose data was obtained from Datastream. Since coupons are paid semi-annually, we choose six-month time interval as a period and set the sampling dates on May 15 and November 15.

Exhibit 1 is the stripped Treasury zero coupon bond prices $v(t)$ on February 7, 2000 given by LP1. We applied pricing equations (2) and (3) in modelling the cash flows and market prices. The last column is the observed US STRIPS prices on the same day from Datastream.

The total error between the market prices and the estimated prices is 2.13 and the total market value of these bonds is 7163 . So the relative error is less than 0.03 percent, a very good fit. The results are exactly the same for several different minimum forward rates $m(t)$ from 0 to 0.03 , which implies the choice of $m(t)$ is fairly robust. Comparing the result with the observed US STRIPS prices we see they are very close.

\section{$3 \quad$ Risky Zero Coupon Bond Prices}

Suppose bonds are classified according to their riskiness into ratings from 1 to $M$ : bond rated 1 has the highest quality and the lowest default risk, and bond rated $M$ has the lowest quality and the highest default risk. Suppose there are $N$ bonds observable on the market. Bond $i$ has present value $P_{i}$, maturity date $T_{i}$, cash flows $c_{i}(t)$ for $t=1,2, \ldots, T_{i}$, and credit rating $d(i)$. Define $c_{i}(t)=0$ for $i=T_{1}+1, \ldots, T$ where $T$ is the longest maturity date among $N$ bonds. Suppose for the class of bonds with credit rating $j$ 
Exhibit 1. US Treasury Zero Coupon Bond Prices

\begin{tabular}{lrrr} 
date & LP1 price & yield (\%) & US STRIPS \\
\hline $15 / 05 / 00$ & .9849 & 5.60 & .9854 \\
$15 / 11 / 00$ & .9539 & 6.12 & .9548 \\
$15 / 05 / 01$ & .9213 & 6.44 & .9215 \\
$15 / 11 / 01$ & .8898 & 6.59 & .8904 \\
$15 / 05 / 02$ & .8604 & 6.62 & .8629 \\
$15 / 11 / 02$ & .8320 & 6.63 & .8363 \\
$15 / 05 / 03$ & .8040 & 6.67 & .8060 \\
$15 / 11 / 03$ & .7791 & 6.62 & .7795 \\
$15 / 05 / 04$ & .7528 & 6.65 & .7526 \\
$15 / 11 / 04$ & .7256 & 6.72 & .7239 \\
$15 / 05 / 05$ & .7017 & 6.72 & .7018 \\
$15 / 11 / 05$ & .6784 & 6.72 & .6789 \\
$15 / 05 / 06$ & .6556 & 6.73 & .6564 \\
$15 / 11 / 06$ & .6350 & 6.71 & .6359 \\
$15 / 05 / 07$ & .6158 & 6.67 & .6136 \\
$15 / 11 / 07$ & .5957 & 6.66 & .5974 \\
$15 / 05 / 08$ & .5785 & 6.62 & .5742 \\
$15 / 11 / 08$ & .5608 & 6.59 & .5560
\end{tabular}


the price of a bond stripped of its coupons paying 1 at date $t$ is $v_{j}(t)$ for $t=1, \ldots, T$. To construct these term structures of spot rate curves of credit risky bonds, assuming we have already calculated the zero coupon Treasury bond prices $v_{0}(t), t=1,2, \ldots, T$, we can formulate and solve the following linear programming problem:

$$
\begin{aligned}
\text { LP2: minimize } & \sum_{i=1}^{N}\left(a_{i}+b_{i}\right) \\
\text { subject to } & P_{i}+a_{i}=\sum_{t=1}^{T} c_{i}(t) v_{d(i)}(t)+b_{i} \\
& v_{j}(t+1)-v_{j+1}(t+1) \geq v_{j}(t)-v_{j+1}(t) \\
& a_{i}, b_{i} \geq 0
\end{aligned}
$$

for $i=1, \ldots, N, j=0, \ldots, M-1$, and $t=0,1, \ldots, T-1$, where $v_{j}(0)=1$.

The inequalities $v_{j}(t+1)-v_{j+1}(t+1) \geq v_{j}(t)-v_{j+1}(t)$ are used to characterize the following properties of bonds: the price of a longer maturity bond is cheaper than that of a shorter maturity bond, and the price of a higher credit rating bond is higher than that of a lower credit rating bond. The first condition is satisfied by rewriting the constraint as $v_{j+1}(t)-v_{j+1}(t+$ $1) \geq v_{j}(t)-v_{j}(t+1)$ and repeatedly applying it from rating $j$ to 0 using the fact that $v_{0}(t)-v_{0}(t+1) \geq 0$. The second condition is satisfied by repeatedly applying the constraint from time 0 to $t$ since $v_{j}(0)-v_{j+1}(0)=1-1=0$. The constraint actually conveys more information: it says that the forward rates of higher credit rated bonds are lower than those of lower credit rated bonds. This will become clear when we study default probabilities of credit risky bonds later in the paper.

We downloaded the list of US industry corporate bonds on 7 February 2000 from the Datastream, which provides information on S\&P rating, amount issued, amount outstanding, next call date, last date price changed, etc as well as all standard bond information. We used 26 AA bonds, 32 A bonds, and 32 BBB bonds with maturity up to 15 November 2005 (six years) after excluding bonds which have no credit ratings, or have call options embedded, or have different issuing amount and outstanding amount, or have no trading for at least two months, or have market value less than 100,000. The last two criteria try to remove bonds whose prices may be irrelevant because of the illiquidity. We have not include AAA, BB, B bond either since there are relatively few such bonds available.

Exhibit 2 is the corporate pure discount bond prices derived using the linear programming model with the Treasury pure discount bond prices as 
Exhibit 2. Risky Zero Coupon Bond Prices and Yield Spreads

\begin{tabular}{l|r|rrr|rrr} 
date & Treasury & AA & A & BBB & AA & A & BBB \\
\hline $15 / 05 / 00$ & .9849 & .9831 & .9831 & .9828 & 67 & 67 & 76 \\
$15 / 11 / 00$ & .9538 & .9478 & .9478 & .9476 & 82 & 82 & 85 \\
$15 / 05 / 01$ & .9214 & .9153 & .9134 & .9109 & 52 & 68 & 90 \\
$15 / 11 / 01$ & .8898 & .8837 & .8818 & .8748 & 38 & 51 & 95 \\
$15 / 05 / 02$ & .8604 & .8503 & .8483 & .8414 & 52 & 62 & 98 \\
$15 / 11 / 02$ & .8320 & .8122 & .8103 & .8034 & 87 & 96 & 127 \\
$15 / 05 / 03$ & .8040 & .7842 & .7823 & .7753 & 76 & 84 & 111 \\
$15 / 11 / 03$ & .7791 & .7551 & .7531 & .7462 & 83 & 90 & 115 \\
$15 / 05 / 04$ & .7528 & .7252 & .7212 & .7143 & 87 & 100 & 123 \\
$15 / 11 / 04$ & .7256 & .6980 & .6940 & .6871 & 81 & 93 & 114 \\
$15 / 05 / 05$ & .7017 & .6737 & .6697 & .6545 & 77 & 88 & 132 \\
$15 / 11 / 05$ & .6784 & .6386 & .6346 & .6194 & 105 & 115 & 158
\end{tabular}

reference $v_{0}(t)$. The last three columns are the yield spreads between the Treasury bonds and the corporate bonds (in basis points).

The increasing gap between riskfree and risky bond prices indicates the increasing default risks over longer terms. The relative errors of LP prices and observed market prices are 0.25 percent for AA bonds, 0.41 percent for A bonds, and 1.16 percent for BBB bonds. The increased errors may partly due to the ripple effects of higher rating bond pricing errors.

We do not need to calculate the Treasury bonds and the corporate bond prices separately, but can calculate their zero coupon prices in the same LP problem. This incorporates the constraints of LP1 and LP2 into

$$
\begin{aligned}
\text { LP3: minimize } & \sum_{i=1}^{N_{0}+N}\left(a_{i}+b_{i}\right) \\
\text { subject to } & P_{i}+a_{i}=\sum_{t=1}^{T} c_{i}(t) v_{d(i)}(t)+b_{i} \\
& v_{0}(t) \geq(1+m(t)) v_{0}(t+1) \\
& v_{j}(t+1)-v_{j+1}(t+1) \geq v_{j}(t)-v_{j+1}(t) \\
& a_{i}, b_{i} \geq 0
\end{aligned}
$$

for $i=1, \ldots, N_{0}+N, j=0,1, \ldots, M-1$, and $t=0,1, \ldots, T-1$. 


\section{Liquidity Issues}

In section 2 we presented a linear programming model to derive pure discount bond prices. The objective is to minimize the sum of all under/over errors. Such a formulation indicates all bonds are treated equally. However the amount issued by each bond may be quite different, from hundreds of thousands of dollars for a corporate bond to tens of millions of dollars for a Treasury bond. This has a significant impact on the liquidity of individual bond. If the amount outstanding of a bond is small, then to compensate for possible illiquidity, the bid ask spread tends to widen which may result in higher/lower bond price in comparison with other more liquid bonds. Therefore we should treat each bond differently according to its liquidity.

One way to do it is to use the amount outstanding information of all bonds in the market, which is readily available from some financial information services, such as the Datastream. If some bonds have much lower amount outstanding than other bonds, we may treat them as illiquid and remove them from the data set. This approach is easily implemented by setting a threshold value and removing any bonds whose amount outstanding is below that value. This is the method we used in the previous two sections. For the Treasury bonds the cutting point is set to be 10 million dollars which is below the amount outstanding of most Treasury bonds. For the corporate bonds the cutting point is 100 thousand dollars. This approach retains most liquid bonds while removes some possible illiquid bonds.

The disadvantage to the above approach is how to choose a threshold value. This problem can be easily solved be reformulating the LP model. Instead of the simple sum of under/over errors of the objective function, we can use the weighted sum of under/over errors. The weight of a bond is the proportion of its amount outstanding to the total amount outstanding of all bonds in the market. To write out this idea mathematically, suppose there are $N$ bonds to be used to derive pure discount bond prices and bond $i$ has amount outstanding $M_{i}$, then the objective function is defined as

$$
\operatorname{minimize} \sum_{i} w_{i}\left(a_{i}+b_{i}\right)
$$

where weights $w_{i}=M_{i} / M$ and $M=M_{1}+\cdots+M_{N}$. The obvious advantage of this approach is that we do not need to set a threshold value to remove possible illiquid bonds. If a bond has smaller amount outstanding, then its weight is also small compare with other bonds. Since weights act as penalty costs in the objective function, the LP model will try to minimize errors of those bonds with larger weights and pay less attention to those 


\begin{tabular}{|c|c|c|c|}
\hline $\begin{array}{c}\text { Exhibit 3. B } \\
\text { date }\end{array}$ & $\begin{array}{l}\text { 3ond Prices } \\
\text { LP price }\end{array}$ & $\begin{array}{l}\text { Using Weig } \\
\text { weighted LP }\end{array}$ & US STRIPS \\
\hline $15 / 05 / 00$ & .9849 & .9849 & .9854 \\
\hline $15 / 11 / 00$ & .9539 & .9539 & .9548 \\
\hline $15 / 05 / 01$ & .9213 & .9214 & .9215 \\
\hline $15 / 11 / 01$ & .8898 & .8898 & .8904 \\
\hline $15 / 05 / 02$ & .8604 & .8604 & .8629 \\
\hline $15 / 11 / 02$ & .8320 & .8322 & .8363 \\
\hline $15 / 05 / 03$ & .8040 & .8038 & .8060 \\
\hline $15 / 11 / 03$ & .7791 & .7791 & .7795 \\
\hline $15 / 05 / 04$ & .7528 & .7528 & .7526 \\
\hline $15 / 11 / 04$ & .7256 & .7256 & .7239 \\
\hline $15 / 05 / 05$ & .7017 & .7022 & .7018 \\
\hline $15 / 11 / 05$ & .6784 & .6783 & .6789 \\
\hline $15 / 05 / 06$ & .6556 & .6555 & .6564 \\
\hline $15 / 11 / 06$ & .6350 & .6354 & .6359 \\
\hline $15 / 05 / 07$ & .6158 & .6158 & .6136 \\
\hline $15 / 11 / 07$ & .5957 & .5957 & .5974 \\
\hline $15 / 05 / 08$ & .5785 & .5785 & .5742 \\
\hline $15 / 11 / 08$ & .5608 & .5604 & .5560 \\
\hline
\end{tabular}

with smaller weights. This in turn removes the effect of bonds with small amount outstanding.

Exhibit 3 is the Treasury pure discount bond prices using the weighted LP model and all relevant Treasury bonds.

Ninety percent of pricing errors are caused by thirty percent of the most illiquid bonds. The prices derived from the two LP models are remarkably close, which may be due to the fact that a threshold value was used in the original LP model.

\section{Sampling Intervals}

So far we have dealt with six monthly intervals between the sampling dates but we might want to have fine sampling dates for the near future and sparse ones for the distant future. The general pricing equations can be extended to allow for this as follows. For each cash flow $c_{k}$ at time $s_{k}$, we can find two 
adjacent sampling dates $t_{n}$ and $t_{n+1}$ such that $s_{k}$ lies in between. Define

$$
\alpha_{k}=\frac{t_{n+1}-s_{k}}{t_{n+1}-t_{n}} .
$$

Then the discount factor $\tilde{v}_{k}$ at time $s_{k}$ can be approximated as

$$
\tilde{v}_{k}=\alpha_{k} v_{n}+\left(1-\alpha_{k}\right) v_{n+1} .
$$

The present value of all cash flows is the sum of $c_{k} \tilde{v}_{k}$, which then leads to a pricing equation.

For example, suppose the sampling periods are six month for the first five years, and then one year for the next ten years, etc. If a bond has three years to maturity, then no change is required. If a bond has ten years to maturity, then in the first five years, the contributions of each cash flow to its adjacent sampling dates are $1-\alpha$ and $\alpha$, respectively. From year six there are two cash flows in each interval, the contributions of the first cash flow to its adjacent sampling dates are $(1+\alpha) / 2$ and $(1-\alpha) / 2$, respectively; while those of the second cash flow are $\alpha / 2$ and $(2-\alpha) / 2$, respectively. This approach can simplify derivation of discount factors for bonds covering very long periods.

For the same bond data as above, we use semiannual interval for years 2000 to 2003, and annual interval for years 2004 to 2008 . The result is given in Exhibit 4 and is compared with the results using semi-annual intervals everywhere. The results are very similar to the equal sampling period results

\section{Default Probabilities}

The above discussions describe a way of constructing theoretical Treasury and corporate pure discount bond prices from the observed coupon bond prices. The yield spread between Treasury strips and corporate strips represents the premium of several risk factors: default risk, liquidity risk, sector risk, etc. To simplify matters, we assume the yield spread is purely due to default risk. This assumption obviously exaggerates the default risk, but it makes calculation of default probabilities easier and at least it gives the upper bound of the risk perceived by the market.

Suppose the Treasury STRIPS prices $v_{0}(t)$ and the corporate zero coupon bond prices $v_{i}(t)$ are given, where $i$ is the credit rating. If a company defaults before its bond matures, then a proportion $\delta$ (the recovery rate) of the face value, discounted by the remaining years to maturity, is given to bond holders. This is the assumption made by most authors, for example, 
Exhibit 4. Bond Prices Using Varying Sampling Intervals

\begin{tabular}{lrrr} 
date & price & yield $(\%)$ & semiannual price \\
\hline $15 / 05 / 00$ & .9849 & 5.60 & .9849 \\
$15 / 11 / 00$ & .9539 & 6.12 & .9539 \\
$15 / 05 / 01$ & .9213 & 6.44 & .9213 \\
$15 / 11 / 01$ & .8898 & 6.59 & .8898 \\
$15 / 05 / 02$ & .8604 & 6.62 & .8604 \\
$15 / 11 / 02$ & .8320 & 6.63 & .8320 \\
$15 / 05 / 03$ & .8040 & 6.67 & .8040 \\
$15 / 11 / 03$ & .7791 & 6.62 & .7791 \\
$15 / 11 / 04$ & .7256 & 6.72 & .7256 \\
$15 / 11 / 05$ & .6784 & 6.72 & .6784 \\
$15 / 11 / 06$ & .6348 & 6.71 & .6350 \\
$15 / 11 / 07$ & .5967 & 6.64 & .5957 \\
$15 / 11 / 08$ & .5608 & 6.59 & .5608
\end{tabular}

Jarrow and Turnbull (1998). (We assume the recovery rate is the same for all bonds, whether AAA bonds or C bonds, for simplicity. This assumption can be relaxed to make $\delta$ credit rating dependent.) Denote $Q_{k}^{i}$ and $P_{k}^{i}$ to be the cumulative default and survival probabilities of a bond currently rated $i$ at the end of period $k$, respectively, and let $q_{k}^{i}$ and $p_{k}^{i}$ be the marginal default and survival probabilities in period $k$, respectively. Then since if a $t$ maturity zero coupon bond does not default at all it is worth $v_{0}(t)$ while under the above assumption if it does default it is worth $\delta v_{0}(t)$, one has

$$
v_{i}(k)=\left(1-Q_{k}^{i}\right) v_{0}(k)+Q_{k}^{i} \delta v_{0}(k)
$$

or

$$
Q_{k}^{i}=\frac{1}{1-\delta}\left(1-\frac{v_{i}(k)}{v_{0}(k)}\right)
$$

for $k=1,2, \ldots$. The other probabilities can be easily computed using the following relations

$$
\begin{aligned}
P_{k}^{i} & =1-Q_{k}^{i} \\
p_{k}^{i} & =P_{k}^{i} / P_{k-1}^{i} \\
q_{k}^{i} & =1-p_{k}^{i} .
\end{aligned}
$$

for $k=1,2, \ldots$, where $P_{0}^{i}=1$, i.e., a risky bond is not in default at time 0 . 
Exhibit 5. An Example by Jarrow and Turnbull (1998)

\begin{tabular}{rrrrr}
$k$ & $v_{0}(k)$ & $v_{i}(k)$ & $q_{k}^{i}$ & $Q_{k}^{i}$ \\
\hline 1 & .953921 & .950486 & .0060 & .0060 \\
2 & .906264 & .897056 & .0110 & .0169 \\
3 & .857820 & .841008 & .0160 & .0327
\end{tabular}

\begin{tabular}{|c|c|c|c|c|c|c|}
\hline Prhibit & Ds & & & & & \\
\hline date & AA & A & $\mathrm{BBB}$ & AA & A & BBB \\
\hline $15 / 05 / 00$ & .0030 & .0030 & .0035 & .0030 & .0030 & .0035 \\
\hline $15 / 11 / 00$ & .0075 & .0075 & .0075 & .0105 & .0105 & .0109 \\
\hline $15 / 05 / 01$ & .0004 & .0040 & .0081 & .0109 & .0144 & .0189 \\
\hline $15 / 11 / 01$ & .0004 & .0005 & .0091 & .0113 & .0149 & .0278 \\
\hline $15 / 05 / 02$ & .0083 & .0084 & .0089 & .0195 & .0232 & .0365 \\
\hline $15 / 11 / 02$ & .0202 & .0204 & .0208 & .0393 & .0431 & .0566 \\
\hline $15 / 05 / 03$ & .0014 & .0016 & .0020 & .0407 & .0446 & .0585 \\
\hline $15 / 11 / 03$ & .0105 & .0106 & .0111 & .0508 & .0548 & .0690 \\
\hline $15 / 05 / 04$ & .0097 & .0143 & .0149 & .0600 & .0683 & .0828 \\
\hline $15 / 11 / 04$ & .0023 & .0027 & .0032 & .0622 & .0708 & .0858 \\
\hline $15 / 05 / 05$ & .0031 & .0034 & .0238 & .0650 & .0740 & .1075 \\
\hline $15 / 11 / 05$ & .0313 & .0316 & .0336 & .0943 & .1033 & . 1375 \\
\hline
\end{tabular}

Exhibit 5 lists default probabilities derived with equations (13) and (14) for an example in Jarrow and Turnbull (1998). The recovery rate $\delta$ is assumed to be 0.4 .

The result is the same as that Jarrow and Turnbull (1998) derived by building an interest rate tree as well as a default tree. The significance of these recursive formulas is twofold: it provides a quick way to compute default probabilities, and it illustrates the independence between default probabilities and interest rate models. The two issues are decoupled.

For the Treasury and corporate zero coupon bond prices derived in section 2 and 3 we can quickly compute the cumulative and marginal default probabilities. The result is listed in Exhibit 6, where columns 2 to 4 are marginal default probabilities and columns 5 to 7 are cumulative default probabilities.

Marginal or cumulative default probabilities which are negative or greater than 1 clearly indicates that there were mispricings on the zero coupon bond prices. The LP formulation discussed earlier ensures that this will not happen. 


\section{Conclusion}

We have shown in the paper that linear programming can be used to strip coupons for both Treasury and corporate bonds. The advantage of the LP approach is that there is no mispricing and the spread structure is built into the model. Real data can be easily analyzed since the LP formulation works whatever the current date, coupon dates, and sampling dates. The weighted LP model can be used to deal with data which may contain some less liquid bonds. Finally default probabilities of risky bonds perceived by the market can be easily calculated without relying on any interest rate models.

\section{Appendix}

Proof of Equations (2) and (3).

There are only two cases need to be considered: 1 . there is no coupon payment between now and the next sampling date, and 2. there is coupon payment between now and the next sampling date. The sampling dates (from the next one) are labelled as $t=1,2, \ldots, T$.

The first case corresponds to $\alpha \geq \beta$. The present value of all cash flows of a bond is equal to sum of $\tilde{c}(t) \tilde{v}(t)$ over all $t$ from 1 to $T-1$, where $\tilde{c}(t)$ is the cash flow and $\tilde{v}(t)$ is the discount factor at time $t$. Using linear interpolation we can write the value of $\tilde{v}(t)$ as a combination of repayments at $t$ and $t+1$

$$
\tilde{v}(t)=\alpha v_{t}+(1-\alpha) v_{t+1}
$$

for $t=1, \ldots, T-1$. Therefore the principal value PV satisfies

$$
\begin{aligned}
\mathrm{PV} & =\tilde{c}(1) \tilde{v}(1)+\cdots+\tilde{c}(T-1) \tilde{v}(T-1) \\
& =c\left(\alpha v_{1}+(1-\alpha) v_{2}\right)+\cdots+(c+F)\left(\alpha v_{T-1}+(1-\alpha) v_{T}\right) \\
& =\alpha c v_{1}+c v_{2}+\cdots+c v_{T-2}+(c+\alpha F) v_{T-1}+(1-\alpha)(c+F) v_{T}
\end{aligned}
$$

The accrued interest is taken in the market to be $(\alpha-\beta) c$ and the general equation for bond prices is given by

$$
\mathrm{MP}+\mathrm{AI}=\mathrm{PV}
$$

where MP is the market (clean) price of the bond. Substituting the accrued interest and the present value into the equation, we obtain the first pricing equation.

The second case corresponds to $\alpha<\beta$. The present value of all cash flows of a bond is equal to sum of $\tilde{c}(t) \tilde{v}(t)$ over all $t$ from 0 to $T-1$. The 
discount factors $\tilde{v}(t)$ can be computed the same as above for $t=1, \ldots, T-1$. In computing $\tilde{v}(0)$, however we must remember that the time interval from now to the first cash flow is $\beta-\alpha$ and the time interval from now to the next sampling date is $\beta$, so linear interpolation gives

$$
\tilde{v}(0)=\frac{\alpha}{\beta}+\frac{\beta-\alpha}{\beta} v_{1} .
$$

If there is only one cash flow in the future, then

$$
\mathrm{PV}=\frac{\alpha}{\beta}(c+F)+\frac{\beta-\alpha}{\beta}(c+F) v_{1} .
$$

If there are two cash flows in the future, then

$$
\mathrm{PV}=\frac{\alpha}{\beta} c+\left(\frac{\beta-\alpha}{\beta} c+\alpha(c+F)\right) v_{1}+(1-\alpha)(c+F) v_{2} .
$$

If there are three or more cash flows in the future, then

$$
\begin{aligned}
\mathrm{PV}= & \frac{\alpha}{\beta} c+\left(\frac{\beta-\alpha}{\beta}+\alpha\right) c v_{1}+c v_{2}+\cdots \\
& +c v_{T-2}+(c+\alpha F) v_{T-1}+(1-\alpha)(c+F) v_{T} .
\end{aligned}
$$

The accrued interest is equal to $(1-\beta+\alpha) c$. Substituting everything into the general equation for bond price gives the second pricing equation.

\section{References}

Carleton, W., and I. Cooper. "Estimation and Uses of the Term Structure of Interest Rates." Journal of Finance, 31, No. 4 (1976), pp. 10671083.

Fabozzi, F. Valuation of Fixed Income Securities and Derivatives. Frank J. Fabozzi Associates, 1998.

Jarrow, R., and S. Turnbull. "Credit Risk." Risk Management and Analysis, ed. Alexander, C., John Wiley \& Sons, 1998, pp. 237-254.

Jarrow, R., D. Lando, and S. Turnbull. "A Markov Model for the Term Structure of Credit Risk Spreads." Review of Financial Studies, 10, No. 2 (1997), pp. 481-523. 
Thomas, L., D. Allen, and N. Morkel-Kingsbury. "A Hidden Markov Chain Model for the Term Structure of Bond Credit Risk Spreads." Capital Markets, Archives of Vols 1-5, 1994-98 (WPS), Vol. 5, No. 26. Working paper, Centre for Financial Markets Research, University of Edinburgh, UK, 1998. 